\section{Temporal variation of seismic parameters in the western part of the India-Eurasia plate collision zone}

\author{
loannis Baskoutas,' \\ George Popandopoulos, ${ }^{2}$ \\ Prasanta Chingtham ${ }^{3}$
}

'Institute of Geodynamics, National

Observatory of Athens, Athens, Greece;

${ }^{2}$ Earthquake Planning and Protection

Organization, Seismotect. Div., Athens,

Greece; ${ }^{3}$ Ministry of Earth Sciences,

Mahasagar Bhavan, Delhi, India

\section{Abstract}

We examined the temporal seismicity variation in the north-west Himalayas and the adjacent regions in relation to strong earthquake occurrences in the period 1970-2010. The aim was to promote seismic hazard assessment and to show the possibilities of strong earthquake forecasting by means of the FastBEE computer tool. The temporal variation of the seismicity is expressed in terms of three basic seismic parameters: the logarithm of the number of earthquakes $\log N$, the seismic energy released in the mode $\log \mathrm{E}^{2 / 3}$ and the b-value of the earthquake magnitude-frequency distribution expressed by the Gutenberg-Richter relation. Significant changes to relative mean values, forming consecutive relative minima and maxima, of the obtained temporal variation series of the seismicity parameters can be considered anomalies. These anomalies were investigated before strong (magnitude $M w \geq 5.6$.) earthquake occurrences and were successfully correlated with 12 strong earthquakes. The mean time of the duration of the anomalies before the origin time of the impending earthquake were estimated to be equal to $3.3 \pm 1.3$ years. We conclude that, in the region under study, the established correlations can be useful for the intermediate-term forecasting of strong earthquakes and that the continuous monitoring of the temporal evolution of seismicity by means of the FastBEE tool can contribute to the evaluation of the seismic hazard status in a target area. The available earthquake data and the results obtained indicate that after the beginning of 2006, the temporal variation of the seismicity does not present clear prognostic anomalies. This behavior is compatible with the absence of earthquakes with a magnitude of $\mathrm{Mw} 6.0$ or more in the area examined.

\section{Introduction}

The north-west Himalayas and adjacent regions, known also as the Central Seismic Gap, ${ }^{1}$ is considered a region of potentially high seismic hazard. It is believed that it could host a great earthquake in the future. In fact, the last big earthquake ${ }^{2}$ of a magnitude range of $M_{W} 7.7$ ore more was experienced nearly 200 years ago, on September 1, 1803. In order to assess the seismic hazard in this area, the temporal variation of seismicity was examined in relation to past strong earthquakes. This investigation was inspired by previous studies, which have shown that spatio-temporal variation of seismicity may help to develop strategies for earthquake forecasting. ${ }^{3-14}$ An exchaustive review of this topic can be found in the recent report by Jordan et al. ${ }^{15}$ For the seismicity analysis, we used the algorithm FastBEE which allows an easy and quick investigation of the temporal variation of the seismicity in a target region FastBEE is suited to obtain time series of three basic seismicity parameters: the logarithm of the non-cumulative number of earthquakes $\log N$, the $b$-value of the earthquake magnitude-frequency distribution as expressed by the Gutenberg-Richter relation, and the seismic energy released in the mode $\log E^{2 / 3} \quad$ Papadopoulos and Baskoutas $^{16,17}$ have shown that this algorithm provides long-term seismic hazard assessment and forecasts probable earthquakes.

Results in the region examined are expected to test the applicability of the FastBEE algorithm in another seismic region of the world, outside Greece, the country where this was initially applied with promising results.

\section{Short description of the seismic parameters}

The temporal variation of the seismicity was obtained through the analysis of the time series of three common seismic parameters: the number of earthquakes, $N(t)$, per time unit, the $b$-value, and the seismic energy.

Estimates of the number of earthquakes per unit time are obtained in the form $\log \mathrm{N}$

$$
\log i(t)=\log \left(\sum_{i=n}^{n i t i} i\right)
$$

where $\mathrm{i}$ is the number of earthquakes, magnitude $M_{W}>M_{\min }, M_{\min }$ is the minimum magnitude of the catalogue completeness in a given area and time interval, $t$ is the time interval of one month, $w$ the length of the smoothing (filter) window, $n(t-w)$ is the number of earthquakes in the smoothing window time interval. The standard error of the calculation is given by the relation $\sigma_{\operatorname{lgN}}=0,4343 / \sqrt{ } \mathrm{N}$

The estimates $\mathrm{b}(\mathrm{t})$ of the $\mathrm{b}$-value were calculated by the maximum likelihood method
Correspondence: Ioannis Baskoutas, Institute of Geodynamics, National Observatory of Athens, Athens, Greece.

E-mail: i.basko@noa.gr; gpapadopoulos@oasp.gr

Key words: temporal seismicity changes, b-value, seismic energy, earthquake forecasting, seismic hazard.

Acknowledgements: the authors would like to thank the Indian Meteorological Department (IMD), New Delhi, for providing the data used in the analysis described in the paper

Received for publication: 1 September 2011. Revision received: 3 November 2011.

Accepted for publication: 11 November 2011.

This work is licensed under a Creative Commons Attribution NonCommercial 3.0 License (CC BYNC 3.0).

(C) Copyright I. Baskoutas et al., 2011

Licensee PAGEPress, Italy

Research in Geophysics 2011; 1:e3

doi:10.4081/rg.2011.e3

and relationship ${ }^{18}$ as follows:

$$
b i f !=\log \left[1+\frac{N_{x}(t-w)}{\sum_{i=}^{n} i-N_{n} \quad w(t-w)}\right] \Delta M
$$

where $\mathrm{N} \Sigma$ is the total number of earthquakes, magnitude $M_{W}>M_{\min }, M_{\min }$ is the minimum magnitude of the catalogue completeness in a given area and time interval, $\mathrm{NM}_{\min +\mathrm{i} \Delta \mathrm{M}}$ is the number of earthquakes in the ith magnitude, $\mathrm{n}=1+\left(\mathrm{M}_{\max }-\mathrm{M}_{\min }\right) / \Delta \mathrm{M}$ is the number of the increment $\Delta \mathrm{M}=0.20$. The standard error of the b-value estimates is obtained by means of the relation $\sigma_{b}(t)=b(t) / \sqrt{ } N_{\Sigma}$

Finally, the parameter $\log \mathrm{E}^{2 / 3}$ which represents the mean seismic energy released in the time unit, is obtained by the relation

$$
\log t^{2}(t)=\log \left(\frac{1}{n(t-w)} \sum_{:=}^{n} t^{2}\right)
$$

where $t$ is the time interval of one month, and $\mathrm{n}(\mathrm{t}-\mathrm{w})$ is the number of earthquakes in the smoothing window time interval. $\mathrm{E}_{\mathrm{i}}$ is the seismic energy of the $i^{\text {th }}$ earthquake in the time window $\mathrm{w}$. The confidence limits were calculated in the range of the examined time period and they were considered a measure of statistical significance.

\section{Temporal variation of regional seismicity}

The temporal variation of the three seismicity parameters was examined by using data taken from the Earthquake Catalogue of the India Meteorological Department (IMD) The 
catalogue comprises 705 events within the rectangular area determined by the geographical coordinates 27-33N and 76-83E. Spatial distribution of hypocenters is shown in Figure 1, while their magnitude time distribution is shown in Figure 2. In Figure 1, the numbered circles represent all strong earthquakes with magnitude Mw 5.6 or more that will be examined retrospectively in relation to the temporal variation of the seismicity. Table 1 reports their catalogue parameters along with the magnitudes from the USGS/NEIC Data Base (Column 8) for a comparison. This catalogue is complete for events of M 4.0 or more in the period 19701980 and fall to the magnitude M 2.5 or more after 1998. Figure 2 shows the time distribution of the magnitudes in the examined period 1970-2009. For the purpose of this study, events with a magnitude of M 4.0 or more were considered in the period 1970-1980 and with M 3.6 or more in the period 190 to 2009. Both magnitude choices are consistent with methodology employed to obtain the qualitative character of the temporal variation estimates.

Figure 3A shows the characteristic FastBEE output of the temporal variation analysis of the seismic parameters, using the IMD seismic catalogue, in the period 1970-2009.

The methodology to obtain such curves is described in detail by Papadopoulos and Baskoutas. ${ }^{16,17}$ Briefly, the estimates of the examined seismic parameters were obtained by a moving window technique, with a step of one month. The moving window length was set as 13 months long. In continuation, the smoothed estimates were re-filtered, with the same order length and triangular filter, in order to avoid side lobs. This procedure makes the temporal changes, with periods equal or greater than the half filter width, pass undistorted 19. This consideration suggests that two or more successive strong events, which their origin time distances less than the filter width, can be associated to the same anomaly.
The FastBEE output shows the temporal variation of the $\log \mathrm{N}$, b-value and $\log \mathrm{E}^{2 / 3}$, from the top to the bottom. Each seismic parameter is expressed as two superposed curves. The first curve (violet thin line) represents the smoothed time series and the second (bolder red line) the filtered one. In the same output, both the standard error of the b-value and the confidence limits, $1 \sigma$, of the parameters $\log \mathrm{N}$ and $\log \mathrm{E}^{2 / 3}$ can be seen as lines parallel to the relative mean. These mean values always refer to the examined time interval. The origin times of the strong events, reported in Table 1, can be seen as numbered arrows perpendicular to the time axis. The serial number of the earthquakes (first column of Table 1) appears

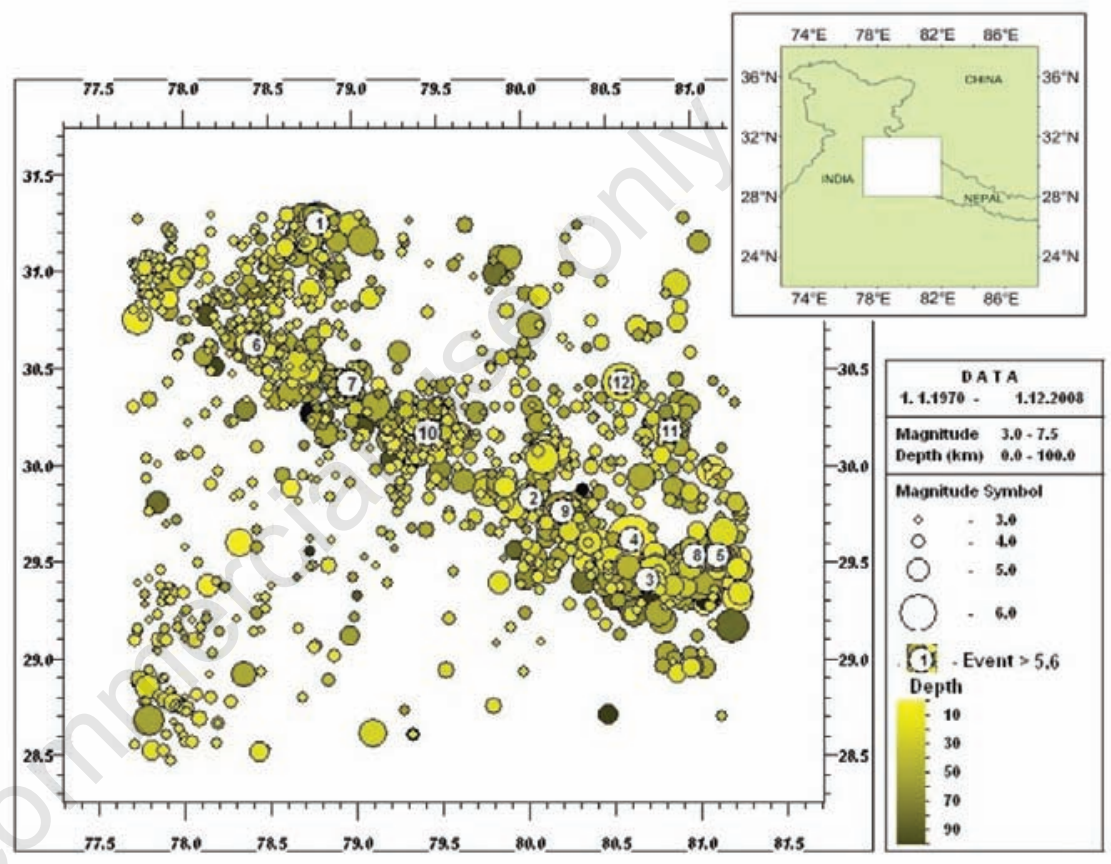

Figure 1. Map of seismic epicenters in the northwest Himalaya and adjacent regions, in the period from 1970 to 2009 . Numbers show epicenters of strong earthquakes of a magnitude of Mw 5.6 ore more. in the upper part of the arrows, while their respective magnitudes (the seventh column of Table 1) in the lower part.

If the origin times of two or more strong events are very close, only the last event's serial number can appear on the output graph because of the time scale. Thus in the case of the Figure 3A event, serial numbers 3 and 7 seem to be missing because they are covered by n. 4 and n. 8 , respectively.

The previously mentioned strong earthquakes, with a magnitude of Mw 5.6 or more, FastBEE graph as a user choice. These events were considered to be the minimum size earthquake occurring in a given area, which are reported in Table 1 and plotted in the

Table 1. Strong earthquakes of Mw 5.6 or more which occurred in the study region in the time period from 1970 to 2009.

\begin{tabular}{|c|c|c|c|c|c|c|c|c|}
\hline S/N & Date & Origin time & Lat & Lon & Dept & $\begin{array}{l}\text { Magn } \\
\text { (IMD) }\end{array}$ & $\begin{array}{l}\text { Magn } \\
\text { (NEIC) }\end{array}$ & $\begin{array}{l}\text { Time anomaly } \\
\text { duration }\end{array}$ \\
\hline 1 & 1975 JAN 19 & 08:12:09.8 & 31.94 & 78.53 & 49 & 5.8 & 6.0 & $\sim 2$ Years \\
\hline 2 & 1979 MAY 20 & $22: 59: 11.5$ & 29.93 & 80.27 & 0 & 5.7 & 5.9 & $\sim 2.8$ Years \\
\hline 3 & 1980 JUL 29 & $12: 23: 07.7$ & 29.34 & 81.21 & 3 & 5.7 & 5.7 & $\sim 4$ Years \\
\hline 4 & 1980 JUL 29 & 14:58:41.6 & 29.63 & 81.09 & 0 & 6.1 & 6.6 & $\sim 3.2$ Years \\
\hline 5 & 1984 MAY 18 & 4:28:52.1 & 29.52 & 81.79 & 0 & 5.6 & 5.6 & $\sim 4.6$ Years \\
\hline 6 & 1986 JUL 16 & 23:03:07.0 & 31.05 & 78.00 & 40 & 5.6 & 6.5 & $\sim 2$ Years \\
\hline 7 & 1991 OCT 19 & 212315.0 & 30.77 & 78.79 & 13 & 6.6 & 6.0 & $\sim 4.5$ Years \\
\hline 8 & 1991 DEC 9 & 1242.0 & 29.51 & 81.61 & 3 & 5.6 & 5.6 & $\sim 4.5$ Years \\
\hline 9 & 1997 JAN 5 & 08:47:25.4 & 29.84 & 80.53 & 33 & 5.6 & 5.6 & $\sim 5$ Years \\
\hline 10 & 1999 MAR 28 & 19:05:13.4 & 30.41 & 79.42 & 21 & 6.8 & 6.8 & $\sim 1.6$ Years \\
\hline 11 & 2002 JUN 4 & 14:36:05.9 & 30.42 & 81.43 & 10 & 5.6 & 5.6 & $\sim 3$ Years \\
\hline 12 & 2004 OCT 26 & 02:11:35.8 & 30.78 & 81.03 & 10 & 5.6 & & \\
\hline
\end{tabular}

IMD, India Meteorological Department; NEIC, National Earthquake International Center. 
can reliably fit with the observed prognostic anomalies of the seismic parameters and thus is defined as Minimum Predictable Magnitude (hereafter MPM). The MPM was determined by visually analyzing the temporal variation series in relation to the past strong earthquake activity in a trial and error iterative procedure. ${ }^{14}$ It is assumed that MPM, depends on the seismotectonic characteristics of a given area and represents, from a physical point of view, the minimum typical response of the medium to the changes of the topic tectonic stress under the constant influence of regional stress fields.

Figure 3A shows a step-wise increment in all parameters, which is more evident in the estimates of the parameters $\log \mathrm{N}$ and $\mathrm{b}$-value. This characteristic also shows the efficiency of the FastBEE tool to qualitatively show the changes in the number of earthquakes with time, as these main changes can be seen quantitatively in Figure 2. In other words FastBEE can depict the quality of the seismic catalogue, prima vista and the seismological network development capability of recording even more seismic events.

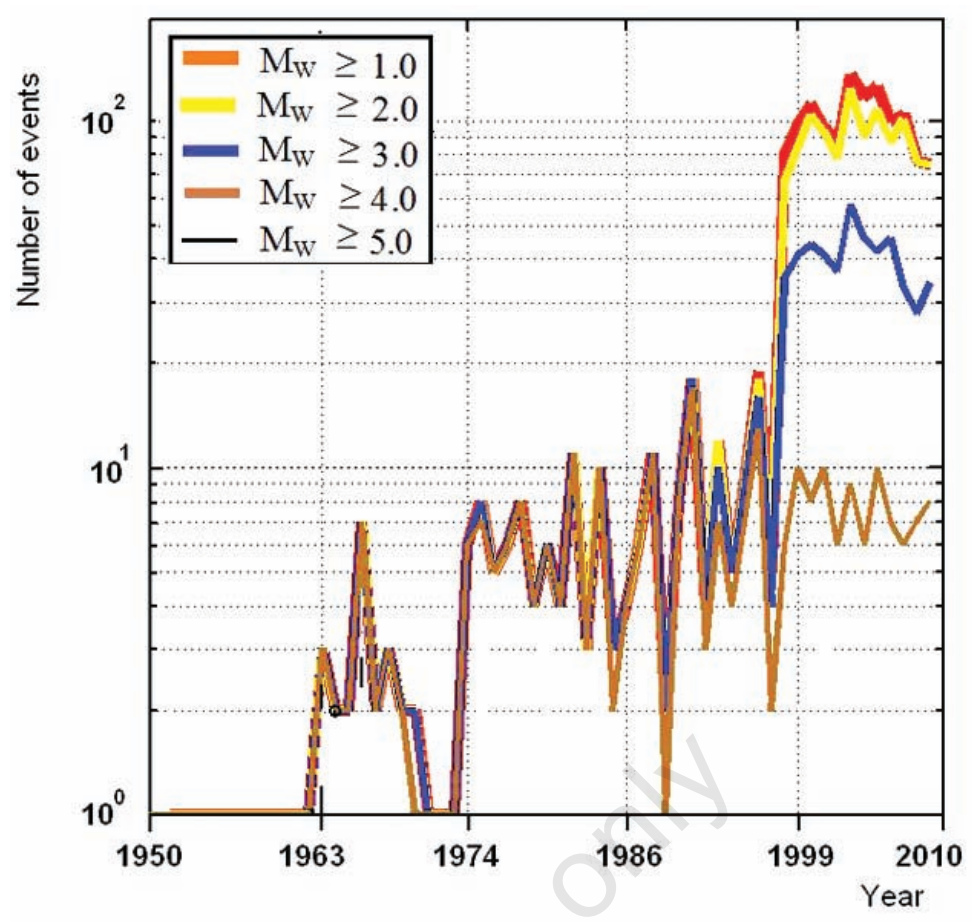

Figure 2. Time distribution of magnitudes in the period 1970-2009.
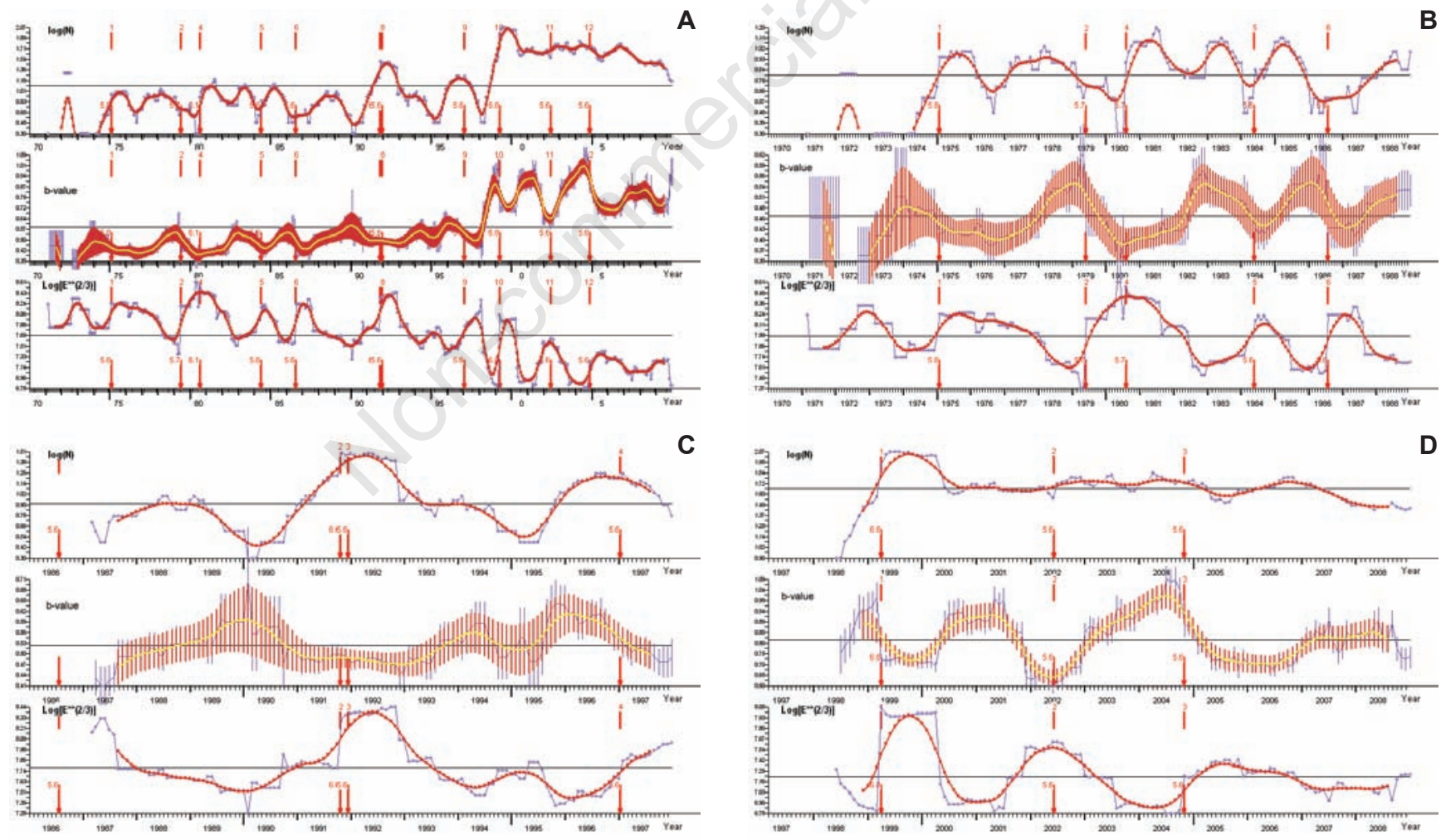

Figure 3. A) Characteristic output of FastBEE analysis, showing the temporal variation of the seismic parameters logN(t), $b$-value with the respective standard errors, and energy released in the form $\log \mathrm{E}^{2 / 3}$. Origin time and magnitude of all strong earthquakes, with magnitude Mw 5.6 ore more are shown as numbered, according to the Table 1 serial numbers, arrows perpendicular to the time axis. B) Temporal variation of the seismic parameters $\log \mathrm{N}(\mathrm{t})$, b-value and energy released in the form $\log \mathrm{E}^{2 / 3}$, with their respective standard errors, in the period 1970-1988. C) Temporal variation of the seismic parameters $\log N(t)$, $b$-value and energy released in the form $\log \mathrm{E}^{2 / 3}$, with their respective standard errors, in the period 1986-1997. D) Temporal variation of the seismic parameters $\operatorname{logN}(t), b-v a l u e$ and energy released in the form $\log \mathrm{E}^{2 / 3}$, with their respective standard errors, in the period 1997-2009. 
The inspection of the temporal variation of $\log \mathrm{N}$ shows that the estimates, in the period 1970-1988, lie below the mean value. After the years 1988 till 2009, these estimates fluctuate clearly above the mean value. For this reason, and for the better representation of the fluctuations of the temporal variation of the seismicity parameters, this period was also subdivided into three sub-periods. The results obtained from data from these new sub-periods, and by using the same analysis parameters, can be seen in Figure 3B, C and D, respectively, which focuses almost exclusively on the sub-periods specified.

Figure 3B-D shows a clear and repeated form of the temporal variation in all three seismicity parameters. These are characterized by consecutive relative minima and maxima, over and above their mean value. The careful visual examination of these figures and especially in the parameters $\log \mathrm{E}^{2 / 3}$ and b-value show that they form mountains and valleys, respectively, which precede almost all the strong earthquakes with magnitude Mw of more than 5.6 (Table 1) which occurred in the area in the examined time period.

The curve of the parameter b-value before all the strong events generally shows an increase stage, starting from a relative minimum toward to a relative maximum, after which it gradually decreases to the mean value and even lower. In general, strong earthquake occurrence is observed during the time period of the decreasing stage. Instead, the curve of the parameter $\log \mathrm{E}^{2 / 3}$, shows a sharp decreasing stage, starting from a relative maximum to a relative minimum, increasing then toward the mean value thus gradually forming a concave shape form with strong earthquakes to occur during the time period of the increasing phase, approaching the mean value. The same temporal behavior, as in the parameter $\log \mathrm{E}^{2 / 3}$, also shows the parameter $\log \mathrm{N}$, although this is not so obvious. The parameter $\log \mathrm{N}$ can usually add information to the evaluation of the anomalies observed in the other two parameters, considering that it reflects the aftershock activity of the preceding strong earthquakes.

From the careful examination of all output figures, it can be seen that the length and sharpness of the prognostic anomalies vary from case to case and from parameter to parameter. Nevertheless, their individual time duration can be measured directly on these graphs. According to Papadopoulos and Baskoutas, ${ }^{16,17} \mathrm{~b}$-value and $\log \mathrm{E}^{2 / 3}$ contain more prognostic information than the parameter $\log N$. For this reason, the durations of the prognostic anomalies, were measured on the b-value and $\log \mathrm{E}^{2 / 3}$ parameter curves. The seismic activity of the region, expressed in terms of $\log \mathrm{N}$, is less informative with respect to the two others, although it can still be used for additional information when recognizing the anomalous periods in the behavior of two other seismic parameters before strong earthquakes. An example is to be found in earthquakes N3 and N4 in Table 1. These events belong to the same, rather delayed prognostic anomaly, starting at the middle of the year 1976. Development is clearer in the b-value and $\log \mathrm{E}^{2 / 3}$ parameters rather than in the $\log \mathrm{N}$ parameter. It means that this pair of earthquakes contaminates the clear development of the temporal anomaly of the parameter $\log \mathrm{N}$ for the event N2.

Results of the temporal variation analysis duration measurements, before the occurrence of the individual, or groups of strong earthquakes in the period 1970-2009, are reported in column 7 of Table 1. These measurements show that their duration ranges from 1.5 to 5 years with a mean value of a smuch as $3.3 \pm 1.3$ years.

Figure 3D shows the characteristic absence of significant variations; in particular, we can see the curve of the temporal variation of the b-value and $\log \mathrm{E}^{2 / 3}$, which ranges around their mean values, in the examined period 20052009 and at the same time the absence of an earthquake with magnitude $\mathrm{M}_{\text {pred }} 5.6$ or more. Nevertheless, as pointed out previously, the continuous monitoring of the temporal variation of the seismic parameters by means of the FastBEE algorithm tool can provide information for the future evaluation of the status of the seismic hazard in this area.

\section{Conclusions}

The temporal variation analysis by means of a set of three seismicity parameters, $\log \mathrm{N}$, bvalue and $\log \mathrm{E}^{2 / 3}$, using FastBEE algorithm, was performed in the northwestern part of the India-Eurasia plate collision zone in the period 1970-2009. The temporal variation profiles obtained of all three examined seismic parameters, show clear fluctuations with repeated characteristic changes around relative mean values which were considered prognostic temporal anomalies. These anomalies were successfully correlated with 12 strong earthquakes, with a magnitude of Mw 5.6 or more, occurring in this area over a 38 -year period of observation. The mean time of the duration of the anomalies before the origin time of the impending earthquake were estimated to be equal to $3.3 \pm 1.3$ years.

The results obtained in this study are similar to those found in Greece, also a very active seismic region, and are equally as promising. $\mathrm{B}$-value and $\log \mathrm{E}^{2 / 3}$ are the most informative parameters for the prediction of strong earthquakes in the context of the FastBEE algorithm, although $\log \mathrm{N}$ in this area has shown much better prognostic behavior than in
Greece area. The results have also revealed the regularity of the temporal variation fluctuation correlation with strong earthquakes of a magnitude of Mw5.6 or more, suggesting that the use of the FastBEE tool is also suitable for this type of analysis in other regions of the world, such as the India-Eurasia collision zone.

According to the seismic data available, the results obtained indicate that after the beginning of the year 2006, the temporal variation of the seismicity does not indicate the presence of clear and suspicious prognostic anomalies showing the absence of the strong earthquake occurrence (magnitude of Mw 5.6 or more). This is compatible with previous findings proving that this behavior indicates the low probability of a strong earthquake occurrence. Nevertheless, the availability of new data, covering the period 2009 till the present, can reveal the seismicity temporal variation behavior in the area; therefore providing an opportunity to assess the seismic hazard for the next couple of years.

\section{References}

1. The Political and Business Daily. "Experts: Uttarakhand faces threat due to seismic zone". Available from: http://www.pbd.in/ index.php?option=com_content\&view=ar ticle\&id=12502: experts-uttarakhandfaces-threat-due-to-seismic-zone\&catid= 8: nation\&Itemid $=6$

2. Ambraseys N, Jackson D. A note on early earthquakes in northern India and southern Tibet. Current Science 2003;84:571-82.

3. Mogi K. Earthquakes and fractures. Tectonophysics 1967;5:35-55.

4. Lukk AA, Nersesov IL. Time Variations in the Parameters of the Seismotectonic Process. Izv Akad Nauk Fiz Zemli 1982;3: 10-27.

5. Varnes DJ. Predicting Earthquakes by Analyzing Accelerating Precursory Seismic Activity. Pure Appl Geophys 1989;130:66186.

6. Knopoff L, Levshina T, Keilis-Borok VI, Mationi C. Increased Long-Range Intermediate-Magnitude Earthquake Activity Prior to Strong Earthquakes in California. J Geophys Res 1996;101:5779-96.

7. Bowman DD, King GCP. Accelerating Seismicity and Stress Accumulation before large Earthquakes. Geophys Rev Lett 2001;28:4039-42.

8. Sobolev GA, Tyupkin YS, Zavyalov AD. Map of Expected Algorithm and RTL Prognostic Parameter: Joint Application. Russ J Earthq Sci 1999;1:301-9.

9. Papazachos B, Papazachos C. Accelerated Preshock Deformation of Broad Regions in the Aegean Area. Pure Appl Geophys 2000; 
157:1663-81.

10. Zavyalov AD. Testing the MME Prediction Algorithm Various Seismically Active Regions in the 1985-2000 Period: Results and Analysis. Izv Phys Earth 2002;38:26275.

11. Keilis-Borok VI, Shebalin P, Gabrielov A, Turcotte D. Reverse Detection of ShortTerm Earthquake Precursors. Phys Earth Planet Inter 2004;145:75-85.

12. Keilis-Borok VI, Soloviev A. Nonlinear Dynamics of the Lithosphere and Earthquake Prediction. Berlin: Springer-Verlag; 2003.

13. Shebalin P, Keilis-Borok VI, Gabrielov A, et al. Short-Term Earthquake Prediction by
Reverse Analysis of Lithosphere Dynamics. Tectonophysics 2006;413:63-75.

14. Baskoutas I, Papadopoulos G, Karakostas V, Papadimitriou E. Recent 2005-2006 strong seismic activity in Greece under the aspect of seismicity parameters temporal variation. Bull Geol Soc Greece 2007;40:1055-62.

15. Jordan ThH, Chen YT, Gasparini P, et al. Operational Earthquake Forecasting: State of Knowledge and Guidelines for Utilization. Ann Geophys 2011;54:316-91.

16. Papadopoulos GA, Baskoutas I. New Tool for the Temporal Variation Analysis of Seismic Parameters. Nat Haz Earth Syst Sci 2009;9:859-64.
17. Papadopoulos GA, Baskoutas I. Regularities in the Time Variations of the Seismic Parameters and Their Implications for Prediction of Strong Earthquakes in Greece. Izv Akad Nauk Fiz Zemli 2011; 11:1-12.

18. Gusev AA. Earthquake prediction from statistical seismicity data, in Seismicity and Seismic Prediction, Upper Mantle Pro-perties and Their Relation to the Kamchatka Volcanism. Nauka Novosibirsk 1974;10919.

19. Bath M. Spectral Analysis in Geophysics. Amsterdam: Elsevier; 1974. 